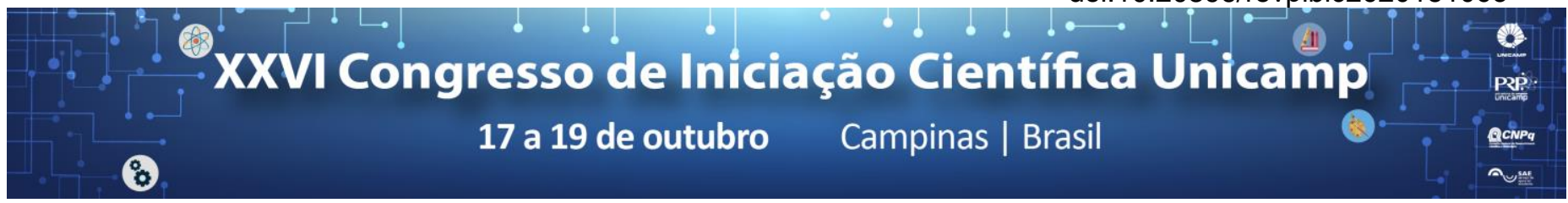

\title{
AVALIAÇÃO DA INFLUÊNCIA DO TRATAMENTO CRÔNICO DE PRAMIPEXOL NA ATIVIDADE LOCOMOTORA EM RATOS SHR.
}

\author{
Nádia Esteves dos Santos*, Camila Aparecida, Beatriz da Silva Franco, Milca Abda, Andrea Maculano Esteves.
}

\begin{abstract}
Resumo
A Síndrome das Pernas Inquietas (SPI) é uma patologia sensoriomotora crônica, com impacto negativo no sono e na qualidade de vida. Ela é caracterizada por sensações anormais nos membros inferiores, com piora noturna e alívio com a movimentação e apresenta relação com 0 envelhecimento. Em seu tratamento, são utilizados agonistas dopaminérgicos, como o pramipexol (PPX). O uso crônico de PPX, ou mesmo o aumento da dose, podem causar um efeito denominado síndrome do aumento (augmentation). O objetivo do presente estudo foi avaliar o efeito do PPX, durante 45 dias em um modelo animal. Foram utilizados ratos SHR com três diferentes idades: com 75 (PPX1 e Salina), 91 (PPX2) e 125 dias de idade (PPX3 e salina). Os animais dos grupos PPX e Salina receberam por 45 dias, no mesmo horário (7AM), uma injeção diária de pramipexol e salina respectivamente. A dose inicial para os grupos PPX1 ( $n=6)$ e Salina $(\mathrm{n}=4)$ foi de com $0,1 \mathrm{mg} / \mathrm{kg}$ nas quatro primeiras semanas, e de $0,25 \mathrm{mg} / \mathrm{kg}$ na quinta e sexta semana. Já os grupos PPX2 $(n=4)$ e PPX3 $(n=4)$, receberam droga nas quatro primeiras semanas de $0,05 \mathrm{mg} / \mathrm{kg}$ e na quinta e sexta semana de $0,25 \mathrm{mg} / \mathrm{kg}$. A avaliação da atividade motora dos animais (teste open field) foi realizada nos momentos basal, e semanalmente antes da administração da droga. Os resultados demonstraram de forma geral que os grupos que receberam PPX tiveram alterações de peso e de comportamento na ambulação periférica, total e rearing em relação ao grupo Salina. No entanto, não foram manifestados sintomas de augmentation durante o tratamento. Nossos resultados mostraram que o tratamento com PPX apresentou alterações no comportamento dos animais após o uso agudo, no entanto, ao avaliar o tratamento crônico, não foi possível verificar os sintomas de augmentation.
\end{abstract}

Palavras-chave: Atividade Locomotora, Pramipexol e Augmentation.

\section{Introdução}

A SPI é uma patologia sensoriomotora crônica, com impacto negativo na arquitetura do sono e na qualidade de vida (Hening WA et al., 2004). O seu diagnóstico é clínico e caracterizado por uma sensação anormal localizada nos membros inferiores, com piora noturna e alívio com a movimentação. Existem alguns agentes terapêuticos com eficácia comprovada por estudos: os agonistas dopaminérgicos (Mathis J, 2005). A piora dos sintomas quando a medicação é usada em longo prazo, chamada de síndrome do aumento (augmentation) (Hening WA et al., 2004). O presente estudo avaliou durante 45 dias de tratamento farmacológico com pramipexol os possíveis sintomas característicos da síndrome de aumento.

\section{Resultados e Discussão}

Os resultados demonstraram de forma geral que os grupos que receberam PPX houve alterações na ambulação periférica, total e rearing. Porém, não foram manifestados sintomas de augmentation ao decorrer do tratamento. Os grupos que receberam PPX apresentaram alteração no peso. Os resultados demonstrados estão expressos em média e erro padrão analisados através do programa Statistic 7. Foi realizado o teste ANOVA medidas repetidas, com post hoc Turkey, e o nível de significância usado foi de $p<0,05$.

Figura 1. Peso dos animais dos grupos (salina, PPX 1, PPX 2 e PPX 3) durante o tratamento com pramipexol e salina.

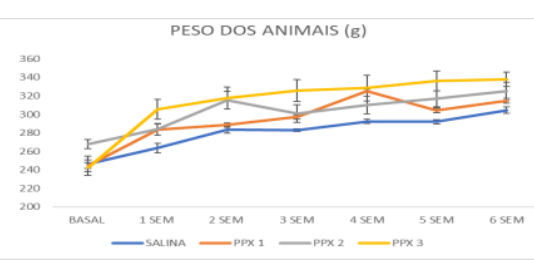

Figura 2: Peso dos animais do grupo Salina e PPX 1 durante o tratamento com pramipexol e salina.

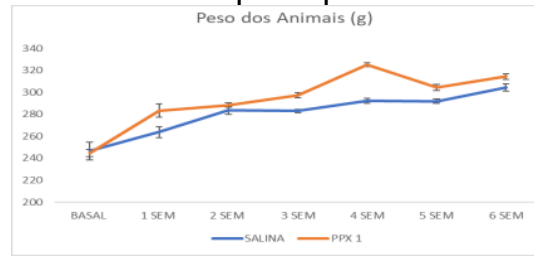

\section{Conclusões}

O tratamento com PPX apresentou alterações no comportamento dos animais após o uso agudo, no entanto, ao avaliar o tratamento crônico, não foi possível verificar os sintomas de augmentation.

\section{Agradecimentos}

Laboratório de Sono e Exercício Físico - UNICAMP e Programa Institucional de Bolsas de Iniciação Científica PIBIC.

HENING WA, Allen RP, Earley CJ, Picchietti DL, Silber MH. Restless Legs Syndrome Task Force of the Standards of Practice Committee of the American Academy of Sleep Medicine. An update on the dopaminergic treatment of restless legs syndrome and periodic limb movement disorder. Sleep; vol. 27, p. 560-583, 2004.

MATHIS J. Update on Restless legs. Swiss Med Wkly,vol. 135, p. $687-$ 696, 2005. 